\title{
THE OVERVIEW OF THE PLANETARY ATMOSPHERIC SPECTRAL TELESCOPE (PAST) IN THE SCIENTIFIC EXPERIMENTAL SYSTEM IN NEAR-SPACE (SENSE)
}

\author{
Q. Meng ${ }^{1}$, F. $\mathrm{He}^{2}$, W. Zhao ${ }^{1}$, K. Wang ${ }^{1}$, L. Yang ${ }^{1}$, J. Dong ${ }^{1}$, X. Wang ${ }^{1}$ \\ ${ }^{1}$ Changchun Institute of Optics, Fine Mechanics and Physics, Chinese Academy of Sciences - (mengqy@ciomp.ac.cn, \\ ciompi@126.com, wangkejun1@163.com, yanglibao228@163.com,dongjihong2002@sohu.com,wangxd@ciomp.ac.cn) \\ ${ }^{2}$ Institute of Geology and Geophysics, Chinese Academy of Sciences - hefei@mail.iggcas.ac.cn
}

KEY WORDS: Telescope, Planetary atmospheric observation, Overall scheme, Two-dimension rotate platform

\begin{abstract}
:
To investigate mass transport and energy dissipation in space environments for solar system planet, a balloon-borne planetary atmospheric spectral telescope (PAST) is designed with $0.8-\mathrm{m}$ aperture in spectral range from $280 \mathrm{~nm}$ to $680 \mathrm{~nm}$ will be floated at 35$40 \mathrm{~km}$ altitude to observe and investigate the global space environment of Mercury, Venus, Mars, and Jupiter. The telescope is designed by Changchun Institute of Optics, Fine Mechanics and Physics, Chinese Academy of Sciences (CIOMP, CAS), and supported by the Strategic Priority Research Program of Chinese Academy of Sciences, that is the Scientific Experimental system in Near-SpacE (SENSE). The telescope is mainly supported by a Ritchey-Chrétien optical system which can achieve 0.5" angular resolution observation, and the optical system has the function of focusing and stabilizing. The telescope is combined with a two-dimension rotate platform to achieve planetary atmospheric imaging in long exposure time. This paper mainly introduces the PAST scheme briefly.
\end{abstract}

\section{INTRODUCTION}

Planetary Atmospheric Plasma Coupling and Comparative Research is a project of the Scientific Experimental system in Near-SpacE (SENSE), and it proposes a global atmospheric remote sensing observation of the atmospheric environment of the near-Earth planet based on the aerostat platform. The designed telescope will be floated on earth atmospheric stratosphere. to observer the thin atmosphere of solar system planets including Venus, Mars, and Jupiter and so on. The observation band covers the ultraviolet to visible light and acquires the main components or characteristic components of the planet. The purpose of the project is to obtain information on the global atmospheric multispectral distribution of the main components or characteristic components of the atmosphere and its evolution information for comparative planetary studies. The research of the subject is expected to make important achievements in revealing the diversity of planetary atmospheric environment evolution and solar energy regulation of the planetary atmosphere.

According to the needs of scientific goals, the PAST which is with a large-aperture, a working spectrum band from ultraviolet to visible was established. PAST will be mounted on an aerostat platform to achieve multispectral imaging of terrestrial planet atmospheres to study the diversity of planetary atmospheric environmental evolution.

\section{PAST SCHEME}

\subsection{Main Technical Index}

According to the task, the PAST main technical index is shown in Table 1.

\begin{tabular}{|c|c|c|}
\hline No. & technical index & value \\
\hline 1 & Aperture $(\mathrm{mm})$ & $800 \mathrm{~mm}$ \\
2 & Field of view & $2 \omega=15^{\prime}$ \\
\hline
\end{tabular}

\begin{tabular}{|c|c|c|c|}
\hline & (FOV) & & \\
\hline 3 & Resolution (") & & 0.5 \\
\hline 4 & $\begin{array}{c}\text { spectral range } \\
(\mathrm{nm})\end{array}$ & $280-680$ & $\begin{array}{l}\text { 280-680 for planetary } \\
\text { atmospheric observation } \\
\text { 400-680 for Planetary sensor }\end{array}$ \\
\hline 6 & $\begin{array}{l}\text { Stray light } \\
\text { coefficient }\end{array}$ & (bey & $\begin{array}{l}\text { Less than } 5 \% \\
\text { ond } 20^{\circ} \text { about optical axis) }\end{array}$ \\
\hline
\end{tabular}

Table 1 The main technical index of PAST

\subsection{Optical System Scheme}

The PAST adopts a catadioptric optical system whose foundation is a Ritchey Chrétien system which has the ability to achieve a well image quality for $15^{\prime}$ FOV with the correcting lenses. In the back focal length, the focusing mirror and stabilizing mirror are set. A beam splitter prism divides the ray path into two parts, one part is for planetary atmospheric spectral imaging (scientific imaging), and the other part is for a planet sensor.

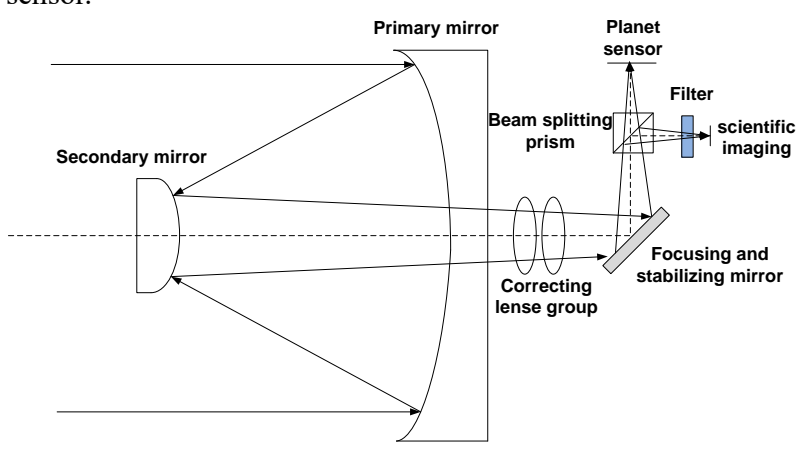

Figure 1 Optical System Scheme 
The focal length of the system is $5600 \mathrm{~mm}$, the relative aperture is $1: 7$, the field of view is $2 \omega=15^{\prime}$, and the working spectrum is $280 \mathrm{~nm}-680 \mathrm{~nm}$. The relative aperture of the primary mirror is $1: 1.75$, the secondary mirror magnification is -4 , the primary mirror (PM) and secondary mirror (SM) distance is $957 \mathrm{~mm}$, and the total length of the system is about $1500 \mathrm{~mm}$.

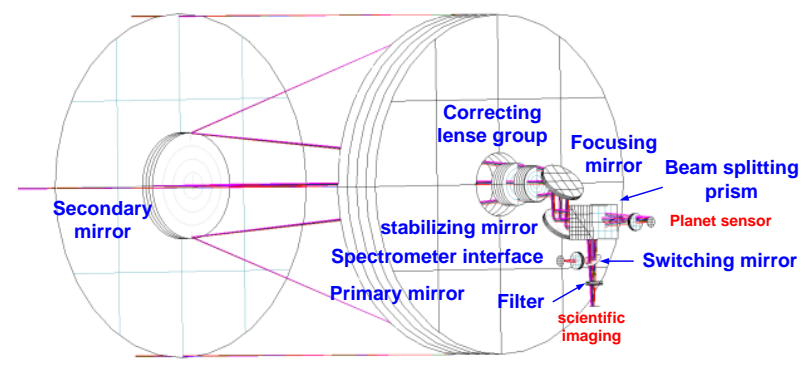

Figure 2 Optical system design result

The optical system can be divided into a front optical portion and a rear optical portion. The front optical portion mainly includes a concave PM and a convex SM, and the remaining optical components are in the rear optical portion, as shown in Figure 2. After the light passes through the PM and SM, the light enters the rear optical portion. In the rear optical path portion, the light first passes through the correcting lens group, and then passes through the two plane mirrors, the former is used for focusing, and the latter is used for stabilization.

The light passes through the cube beam splitting prism and divides the light path into two parts by the functional film layer. The light reflected by the beam splitting prism is used for planetary atmospheric spectral scientific observation and spectrometer imaging (a folder mirror will fold the light into a spectrometer when the planetary atmospheric spectral scientific observation suspends work), and the light transmitted by the beam splitting prism is used to planet sensor.

In the scientific observation and spectrometer imaging optical path, the spectrometer switching mirror (planar mirror) is used to time-separate the scientific observation focal plane and the spectrometer focal plane, shown in Figure 2. A filter wheel is placed in front of the scientific observation focus to achieve selective imaging of different scientific observations. In order to ensure the optical path of each light path is consistent, an optical path compensation sheet is set in front of other focal points.

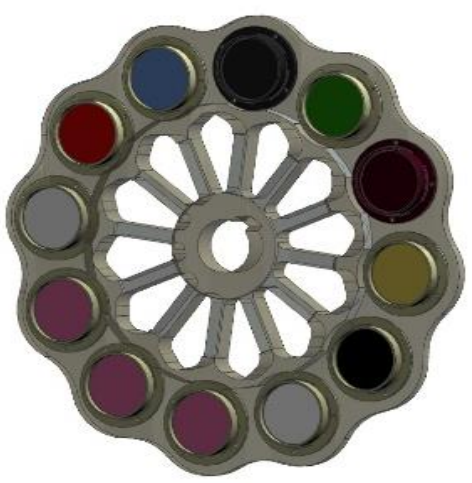

Figure 3 The filter wheel

The point spread function is an important criterion for evaluating star point images. After the optical system is designed, the point spread function is evaluated. In the analysis, the six field of view points of the system are selected, and the optical system point spread function is symmetric in shape and good in energy concentration, shown in Figure 4. The system RMS wavefront error average value is $0.0218 \lambda(\lambda=480 \mathrm{~nm})$, shown in Figure 5 .

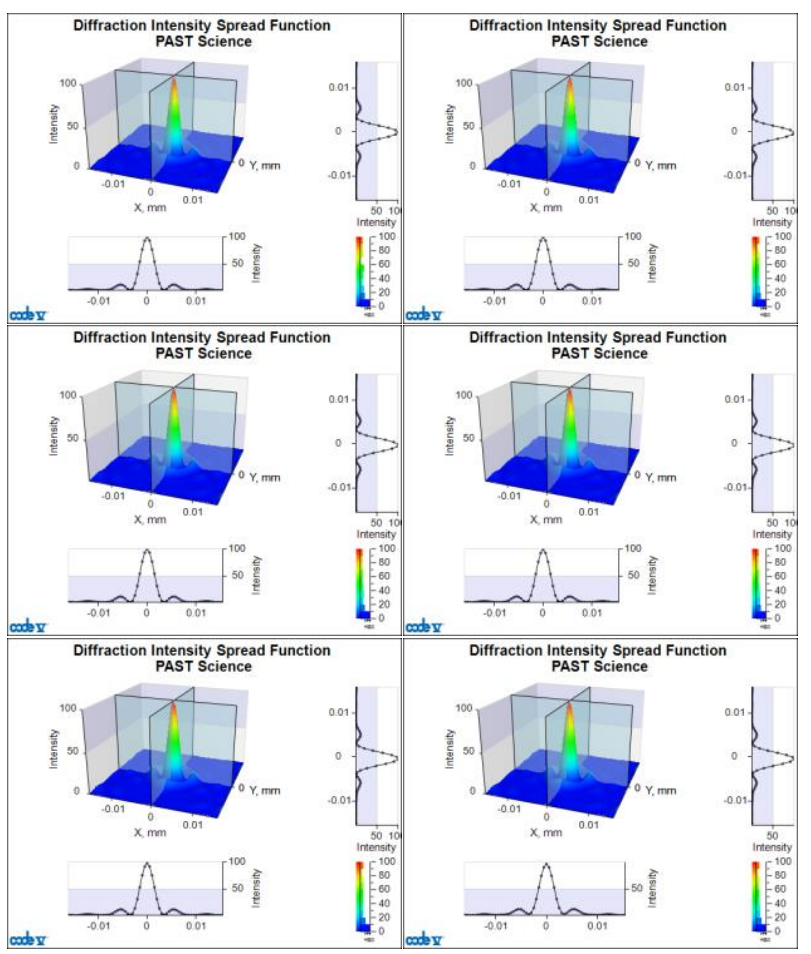

Figure 4 The system point spread function

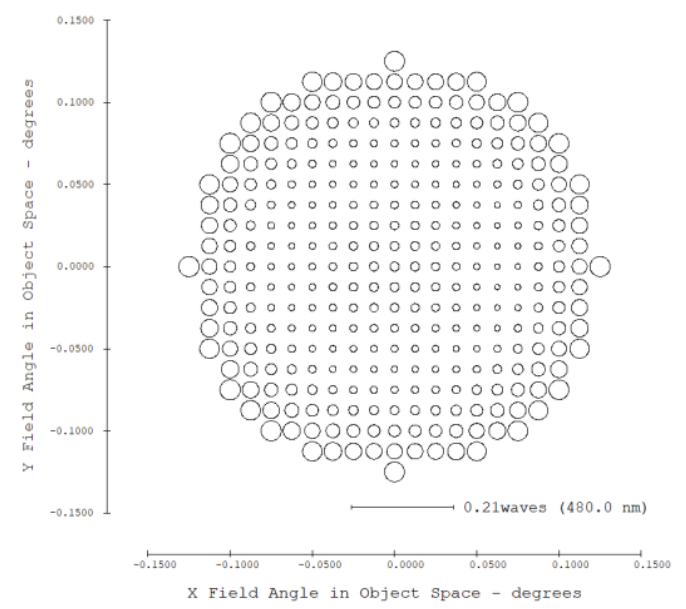

Figure 5 RMS wavefront error

Due to the field of view value is small, the PAST optical system has a good performance in distortion value, the largest value is less than $0.01 \%$, the distortion value is shown in Table 2 , and the distortion curve is shown in Figure 6.

\begin{tabular}{|c|c|}
\hline Relative field of view & Distortion $(\%)$ \\
\hline 0 & 0 \\
0.1 & 0.00008 \\
0.2 & 0.00033 \\
0.3 & 0.00074 \\
\hline
\end{tabular}




\begin{tabular}{|c|l|}
\cline { 2 - 2 } 0.4 & 0.00131 \\
0.5 & 0.00205 \\
0.6 & 0.00295 \\
0.7 & 0.00401 \\
0.8 & 0.00524 \\
0.9 & 0.00663 \\
1 & 0.00819 \\
\hline
\end{tabular}

Table 2 Distortion

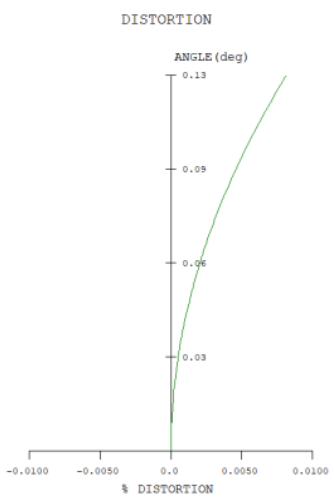

Figure 6 Distortion curve

The optomechanical structure of the telescope includes the stray light suppression structure, includes the hoods, the light blocking rings and diaphragm, etc, shown in Figure 7. The stray light suppression black paint is sprayed inside the optomechanical structure, the absorption rate among the visible spectrum band is large than $95 \%$.

In Tracepro software, rays are traced in all directions beyond $20^{\circ}$, analyzing the stray path outside the field of view, the stray light coefficient is $3.1 \%$, the point source transmission (PST) ratio curve is shown in Figure 8.

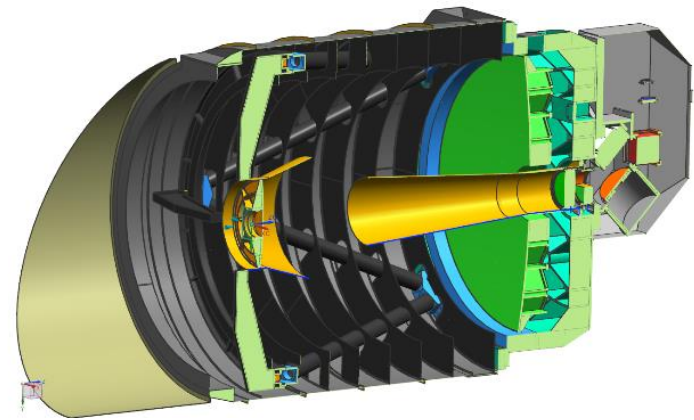

Figure 7 Stray light suppression structure

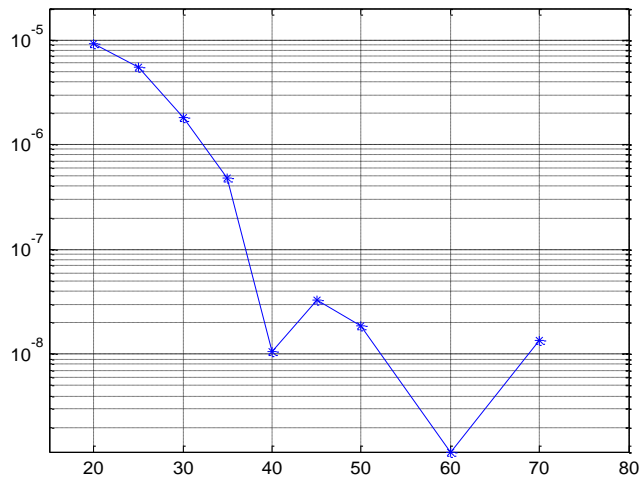

Figure 8 PST curve

\subsection{Opto-Mechanics Scheme}

In the design of the optomechanical structure, we refer to the structure of the Ritchey-Chrétien telescope in Embry-Riddle observatory, and the $2.16 \mathrm{~m}$ telescope at the Xinglong Observatory in Hebei, China, shown in Figure 9. These telescopes are close to PAST in terms of main mirror size and system specifications, and these telescopes are all truss-type structures.

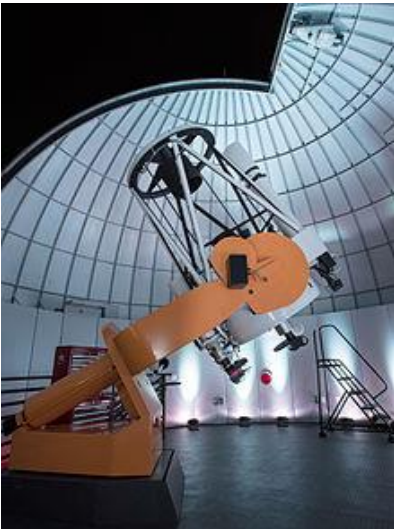

(a)

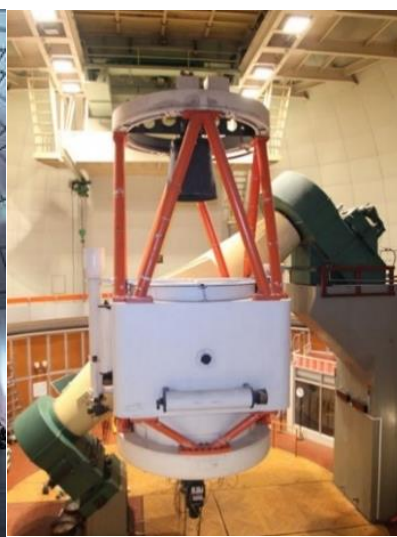

(b)
Figure 9 (a) the Ritchey-Chrétien telescope in Embry-Riddle observatory $\bigcirc$ wikipedia Image Copyright 2019; (b) the $2.16 \mathrm{~m}$ telescope at the Xinglong Observatory in Hebei, China

CNational Astronomical Observatories, Chinese Academy of Sciences Image Copyright 2019

PAST structure is mainly composed of the following parts: PM room, PM structural support, PM support ring, truss rod, SM structural component, SM hood, SM support ring, and spider support and so on, shown in Figure 10. To ensure mechanical properties and reduce system quality, PAST structure is mainly designed with carbon fiber material and titanium alloy material. 


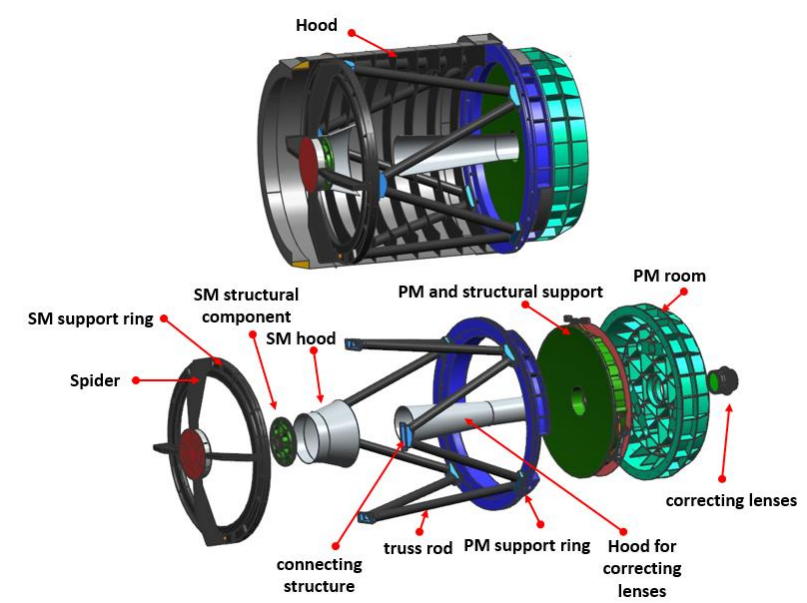

Figure 10 Machinery exploded figure of telescope

The large-aperture mirror surface error performance is the key to static design. Finite element model has been analyzed for the mirror surface shape error in the optical axis horizontal state and the optical axis vertical state, and it shows that the mirror shape error achieves a perfornance value shown in Figure 11.

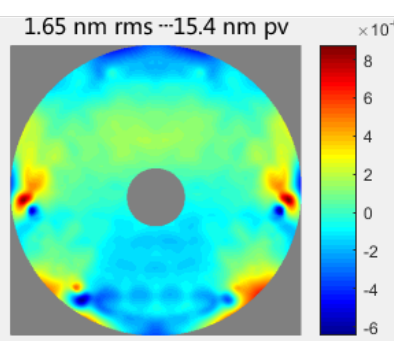

(a) Horizontal optical axis

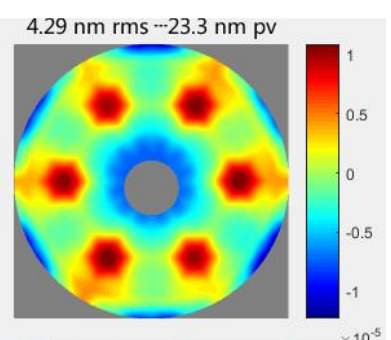

(b)Vertical optical axis
Figure 11 Finite element analysis for mirror shapr error

\subsection{Two-Dimension Rotate Platform Scheme}

Two-Dimension Rotate Platform is chosen from the mature product of Changchun Institute of Optics, Fine Mechanics and Physics, shown in Figure 12. The total weight of the twodimensional rotating platform and telescope is $700 \mathrm{~kg}$. The telescope is placed on a balloon platform which almost $1000 \mathrm{~kg}$.

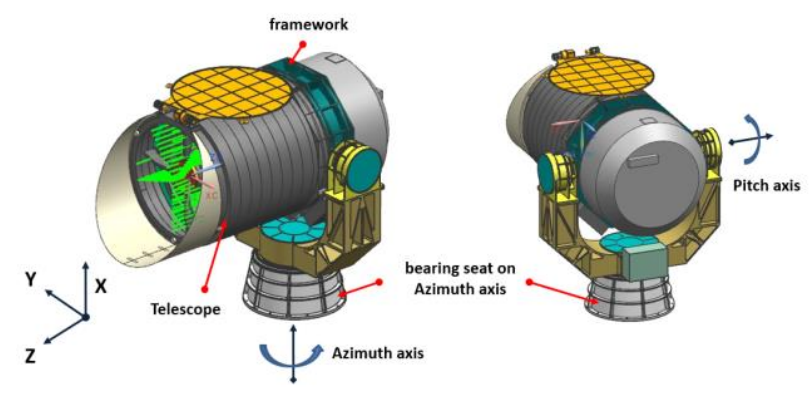

Figure 12 Two-Dimension Rotate Platform Scheme

The structural modal frequencies of the optical axis $0^{\circ}$ and the optical axis $65^{\circ}$ are calculated separately. The first two frequencies of the whole machine are shown in Table 3. The fundamental frequency of the whole machine is $22.7 \mathrm{~Hz}$ in both the optical axis $0^{\circ}$ and the optical axis $65^{\circ}$. The first two modes of the whole machine in different states are shown in Table 3.

\begin{tabular}{|cc|c|}
\hline & Horizontal optical axis & Vertical optical axis \\
\hline $\begin{array}{c}\text { First order } \\
\text { frequency/Hz }\end{array}$ & 22.74 & 22.74 \\
$\begin{array}{c}\text { Second order } \\
\text { frequency/Hz }\end{array}$ & 40.53 & 46.23 \\
\hline
\end{tabular}

Table 3 Vibration frequency of the whole machine under different conditions

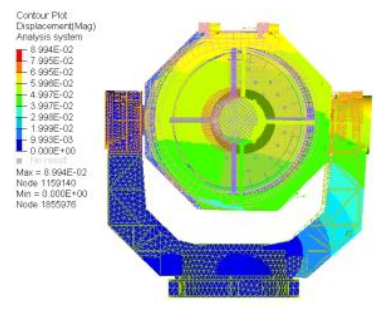

(a) First order mode

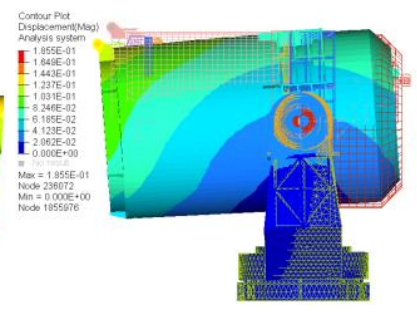

(b) Second order mode

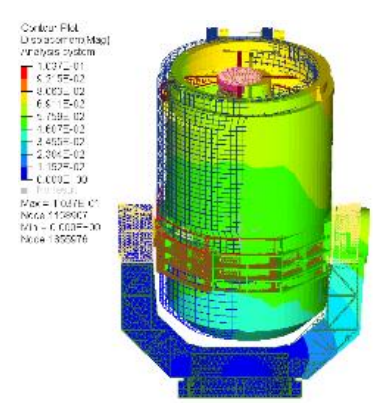

(a) First order mode

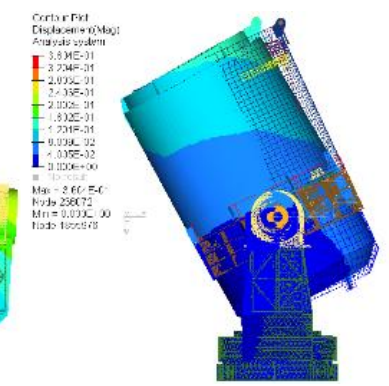

(b) Second order mode
Figure 14 Modal analysis in optical axis $65^{\circ}$

\section{CONCLUSION}

The PAST is mainly composed of a two-mirror catadioptric telescope and a two-dimension rotate platform. It will be floated in 2020 at $35-40 \mathrm{~km}$ altitude earth atmospheric stratosphere to observe and investigate the global space environment of Mercury, Venus, Mars, and Jupiter and so on. Now, the PAST project design has been completed and the manufacture has been started from January 2019. We will release the PAST program progress timely in future.

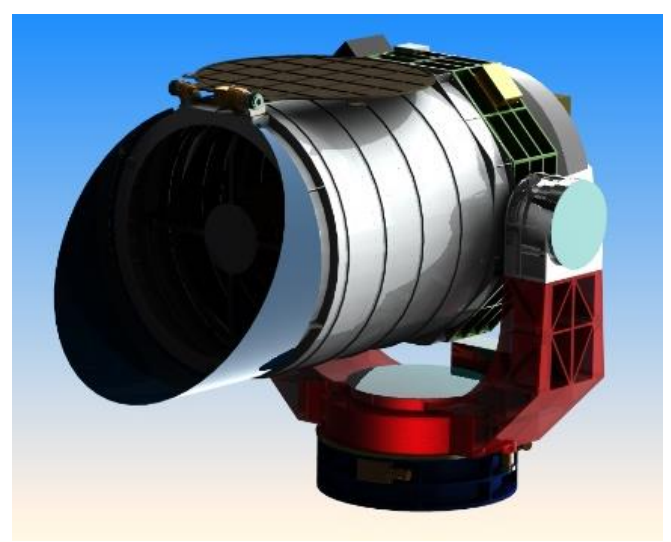

Figure 15 Outside view of PAST 


\section{ACKNOWLEDGEMENTS}

This work was supported by Strategic Priority Research Program of Chinese Academy of Sciences (Grant No. XDA17010205); National Natural Science Foundation of China (NSFC) (Grant No. 61705220); ShuGuang Talents Scheme Award of Changchun Institute of Optics, Fine Mechanics and Physics (CIOMP); Youth Innovation Promotion Association, CAS (2019219).

\section{REFERENCES}

Meng Q., 2014. Easy-aligned off-axis three-mirror system with wide field of view using freeform surface based on integration of primary and tertiary mirror. Applied Optics, 53(14), 30283034

R. N. Wilson.,2007. Reflecting Telescope Optics I. Springer, Berlin Heidelberg, 42-55 